\title{
A Probabilistic Framework for Offshore Wind Turbine Loss Assessment
}

\author{
David Wilkie $^{\mathrm{a} *}$, Carmine Galasso ${ }^{\mathrm{a}}$ \\ ${ }^{a}$ Department of Civil, Environmental \& Geomatic Engineering, University College London, \\ UK \\ *E-mail: david.wilkie.15@ucl.ac.uk
}

\begin{abstract}
During their operational life, offshore wind turbines (OWTs) may be exposed to severe storms, resulting in extreme wind and wave loading acting on the OWT structure (and substructure). This paper proposes quantifying financial losses associated with an OWT exposed to extreme wind and waves using a probabilistic risk modelling framework, as a first step towards evaluating offshore wind farm (OWF) resilience. The proposed modelling framework includes a number of novel elements: 1) the development of site-specific fragility relationships (i.e., likelihood of different levels of damage experienced by an OWT over a range of hazard intensities), properly accounting for uncertainties in both structural capacity and demands; 2) the implementation of a closed-form technique, based on a combinatorial system reliability approach, to assess failure consequences (e.g., financial loss) for both structural and nonstructural components; 3 ) a coherent treatment of epistemic uncertainties across the framework (e.g., sampling variability in fragility estimation), providing loss results accounting for uncertainty of estimation. These aspects can allow for more informative comparisons of various design solutions in terms of structural fragility and risk and/or an improved evaluation of probabilistic losses for decision making. An illustrative application to two case-study sites is presented as a simplified walk-through of the calculation steps in the proposed framework, discussing possible outcomes. For instance, the results from the illustrative application indicate the structural components play an important role in the overall risk profile of an OWT, but this depends on the site-specific wind and wave conditions. The calculation of losses provides a foundation from which a more detailed assessment of OWT and OWF resilience could be developed.
\end{abstract}

Keywords: Offshore Wind; Structural Reliability; Resilience; Loss Modelling; Fragility.

\section{Introduction}

The offshore wind industry has grown to the point where it supplies $12.6 \mathrm{GW}$ [1] of electricity within Europe, with a further $24.6 \mathrm{GW}$ worth of projects due to be installed by 2020 [1,2]. Historically, this form of energy production has been more expensive than others; however, the overall cost of offshore wind farms (OWFs) has recently dropped, driven by reductions in the cost of debt financing. Nevertheless, cost saving remains an important objective for operators who must enter competitive bids for potential OWF sites.

A recent report by the UK Government [3] highlighted that 'integrated design' could potentially contribute to cost reduction. However, achieving improved integrated design is challenging as offshore wind turbines (OWTs) are unique engineering systems, depending on both electrical and mechanical components (such as a generator, gearbox and control system) and structural components (tower, monopile, transition piece and blades) to remain operational. Currently, structural design of OWTs is undertaken at the component level, with the tower and the monopile commonly being designed separately [4]. In this discussion, we focus on OWT supported by monopile foundations [5], as these represent about $80 \%$ of existing and planned offshore wind installations within European waters. Prescriptive, semi-probabilistic approaches are used to evaluate (and compare) potential design alternatives. In particular, current state-of

Towards Resilient OWF: A Probabilistic Framework for OWT Loss Assessment $10 / 06 / 2019$ 
practice codes and standards do not prescribe to explicitly assess structural risk due to uncertainties associated with physical properties of OWTs (e.g., material, geometry) and loading conditions (e.g., wind and wave loading). Instead, they deal with such uncertainties in a conservative way, by employing safety factors to ensure a structure (or a structural component) does not 'fail' with a frequency greater than a pre-defined, target rate. Any 'integrated design' of new OWTs (and integrated assessment of existing ones, for instance, for their life extension) should explicitly account for both uncertainty in environmental conditions, especially those occurring during extreme events (e.g., severe windstorm), as well as the possible complex interdependencies between component-level structural demands and capacities. For instance, stopping the rotor affects the loading on the blades which in turn influences loads on the tower and monopile. The challenge is how to quantify the risk associated with these diverse sub-systems in a harmonized way, accounting for the impact of their failure on the overall structural performance and operability of the asset and farm. Also, since both aleatory and epistemic uncertainty lies at the heart of risk modeling, they require an appreciation at all modeling stages. Thus, a probabilistic approach is the most appropriate one to model the complexity of environmental loading and their impact on an OWT. The concept of resilience provides an effective framework for assessing an OWT as a system of integrated structural and mechanical/electrical components, focusing on the functionality performance of the overall system and not just that of individual components.

The concept of resilience is introduced in Section 2, where it is discussed in relation to the specific challenges encountered in assessing OWT performances. The analytical framework proposed to assess the combined financial losses due to failure of structural and/or mechanical/electrical components is discussed in Section 3. This includes a novel procedure for evaluating failure probability of structural components based on structural fragility analysis employing dynamic structural simulations and advancing uncertainty modelling to derive fragility relationships (i.e., likelihood of different levels of damage experienced by an OWT over a range of hazard intensities). A novel closed-form technique, based on a combinatorial system reliability approach, is employed to assess failure consequences (e.g., financial loss) for both structural and non-structural components. The overall calculation procedure is demonstrated through an illustrative example, where a National Renewable Energy Laboratory (NREL) 5MW OWT is assessed at two wind-farm sites in the Dutch sector of the North Sea, experiencing windstorms (extratropical cyclones), and offshore from the coast of Massachusetts, experiencing hurricane-like conditions. Section 4 introduces the input data and the simplifying assumptions used in the illustrative application of the proposed framework. Finally, Section 5 illustrates the type of results that can be generated through the proposed framework and some specific findings.

\section{Structural resilience of OWTs}

The concept of resilience provides an effective framework for enabling integrated design of a given engineering system, allowing a rational assessment of the system performance in the presence of uncertainties. The term 'resilience' has been applied across a large number of different fields, ranging from ecology, to preparedness of communities exposed to natural hazards and structural design [6]. Therefore, many overlapping definitions for the various components of a resilience assessment framework exist, as summarized by Hosseini et al [7] and Ayyub et al [8], among others. One possible definition suitable for application to structural systems, characterizes resilience through quantifiable metrics [9], also allowing comparison between different systems. According to this definition, resilience can be schematically described through Figure 1, often referred to as the resilience triangle [10]. The solid line indicates the system performance (e.g., in terms of electricity generation, in the case of an OWT) which is reduced after a disruptive event (e.g., a windstorm) occurring at $t_{0}$; the system performance gradually recovers along the time axis, to an 'as-new', 'at-event', or 'deteriorated'

Towards Resilient OWF: A Probabilistic Framework for OWT Loss Assessment $10 / 06 / 2019$ 
performance level. In this context, an operator has different options for repairing the system, depending on specific objectives, including repairing to as-new, at-event or a deteriorate functionality (following the original system deterioration path). Resilience is numerically quantified as the area below the performance curve, and is quantified through the following four metrics which characterizing a given system [11]:

- Robustness - The capability of the system to withstand a disruptive event. It can be quantified as the residual functionality directly after the event occurs and it is therefore a measure of the overall system performance.

- Rapidity - The speed to recover, contain losses, and avoid future disruptions. It can be viewed as the rate of recovery (i.e., the slope of recovery in Figure 1), and therefore determines the time gap from $t_{0}$ to $t_{D}, t_{E}$ or $t_{N}$.

- Redundancy - The extent to which other components can satisfy and sustain functional requirements after a disruptive event causing a loss of functionality.

- Resourcefulness - The capacity to diagnose and prioritize problems that can cause reduced functionality, then to initiate measures that will lead to functional recovery. This relates to the ability of an organization to react after an extreme event has occurred and therefore influences the rapidity.

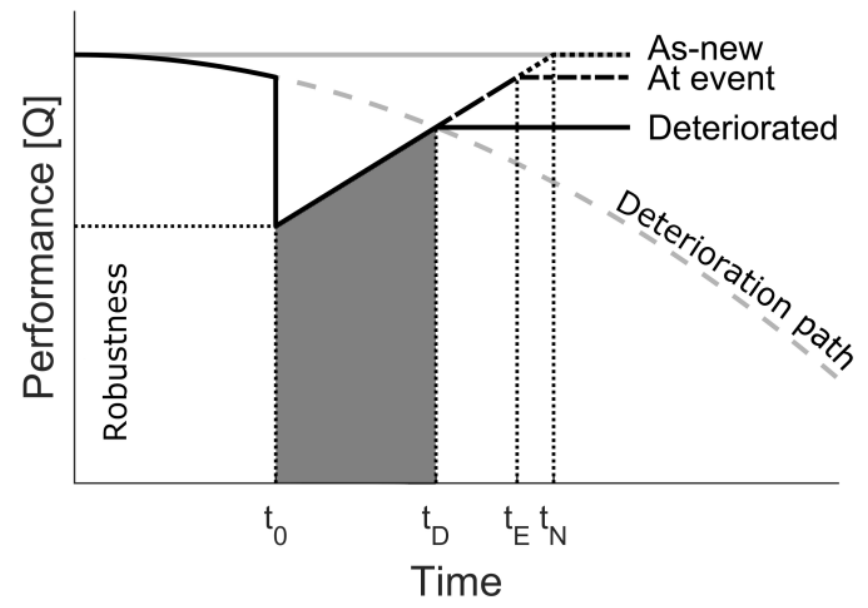

FIGURE 1. Graphical definition of resilience after an event (at $t_{0}$ ), with different repair options (adapted from [11]).

It may be difficult to quantify some of these metrics, especially at the design stage. For instance, information regarding the capacity of an organization to make budget available in the case of a disruptive event (i.e., part of resourcefulness) is seldom available to a design engineer. Nor would it be clear to a designer whether an operator would decide to restore functionality to the original or to a degraded level (i.e., $t_{D}$ in Figure 1). Therefore, a methodology for assessing structural resilience of OWTs relying on its robustness and redundancy features would allow this concept to be directly applied at the design stage. The initial design-stage estimate of an OWT robustness could be used in a full resilience calculation at a later stage which also considered recovery. An approach, investigated by Bruneau and Reinhorn [12] and applied here, assumes that loss of functionality after a disruptive event and the time to recovery are highly correlated. This is intuitive as, in general, if an event (e.g. a windstorm) causes more damage, it will take longer to repair the considered asset. A similar approach has previously been applied to structures experiencing blast [13] by defining a relative resilience indicator $(R R I)$, which is correlated to the overall structural resilience $(R)$ :

$$
R(E) \propto R R I(E)=1 / C(E)
$$

Towards Resilient OWF: A Probabilistic Framework for OWT Loss Assessment 10/06/2019 
In Eq. (1), RRI can be defined as the inverse of the consequence (C) of a disruptive event (E). Under this assumption, a structure experiencing a lower consequence (i.e., less damage and lower financial loss) as the result of a hazardous event, is viewed as more resilient.

This approach is suitable for application to OWTs and OWFs; however, the metric quantifying consequence needs to correctly represent the effect of failure of individual components on the OWT (or OWF) functionality. Considering a single OWT structure, loss of an important subassembly will completely stop production, resulting in the OWT dropping to zero functionality. However, the failure of different components will have different implications in terms of recovery time. Therefore, it is not enough to define consequence using the reduction in functionality caused by component failure alone. This consideration also precludes the use of some common structural consequence measures, such as percentage of the structure collapsing [13], or percentage of mechanical components failing, which do not provide sufficiently detailed information about the type of failure. Other metrics, such as those relating to life-safety are not of primary importance for offshore wind as the turbine is normally unmanned, apart from brief periods to allow for maintenance activities [14]. Rose et al [15] quantified resilience of an OWT through robustness by estimating the cost incurred by loss of functionality after an extreme event. This allows the failure severity of different components to be compared within a unified metric, because each has a different material cost (replacement). Financial loss is also easy to communicate to stakeholders and is therefore used in this study. However, it neglects the operational costs of repair, such as hiring vessels, which are expensive but difficult to quantify.

\section{$3 \quad$ Probabilistic risk modelling framework for OWTs}

A probabilistic risk modelling framework is proposed here to assess structural and, potentially, non-structural risk associated with OWTs exposed to extreme environmental conditions. It is based on decomposing the total risk into conditional probability distributions which are evaluated sequentially (and independently), and finally combined using the law of total probability. Similar approaches have been applied to assess structures exposed to seismic [16], wind [17,18], tsunamis [19] and blast [13] hazards, among others. It is worth noting that this approach is often referred to as catastrophe risk modelling, when the focus in on portfolios of assets for (re-)insurance purposes, or performance-based design/assessment, focusing on individual assets for design/retrofitting purposes. The proposed framework outputs the annual rate of exceedance of a given loss level, $L,(\lambda(L))$, as a function of various complementary cumulative distribution functions (CCDFs; or $G[\cdot \mid \cdot])$ :

$$
\lambda(L)=\iint G[L \mid D S] \cdot|d G[D S \mid I M]| \cdot|d \lambda(I M)|
$$

In Eq. (2), the main interface variables are: a measure of the intensity of a natural hazard (intensity measure; or IM), e.g., wind speed or wave height; and damage states (DSs), e.g., the performance level of the structure and/or its components, as a function of the given IM. This framework can be schematically represented through the flowchart in Figure 2, where the individual tasks include:

- Hazard analysis - assessing (extreme) conditions that have the potential to cause damage to the system being assessed. The hazard analysis predicts the probability distributions of these conditions occurring based on measured site data or physicsbased models, for instance based on large catalogues of simulated events capturing the frequency, severity, location, and other characteristics of the entire spectrum of plausible real hazardous events

Towards Resilient OWF: A Probabilistic Framework for OWT Loss Assessment 
- Exposure (or structural) characterization - characterizing the properties of the structure and the value at risk, including information about location, construction details (e.g., structural geometry), and replacement costs.

- Fragility analysis - assessing the probability of damage occurring conditioned on the hazard intensity. This also allows to account for the uncertainty in structural capacity (e.g., geometry, material properties, and models) and sampling.

- Loss analysis -estimating financial losses, providing metrics that can be used to assess the performance of the system.

Structural and equipment components need to be treated differently because structural failure is usually predicted analytically (or numerically), based on structural simulations, whereas equipment failure data can be usually derived from empirical databases, mainly recording the rate of failure, usually without (any) reference to specific environmental conditions. Consequently, equipment failure is not typically conditional on the IM and does not require site-specific analysis. The general probabilistic risk modelling framework shown in Figure 2 can be adapted to the specific loss analysis of an OWT (Figure 3), considering both structural and mechanical/electrical equipment. The elements of Figure 3 are described in more detail in the following sub-sections.

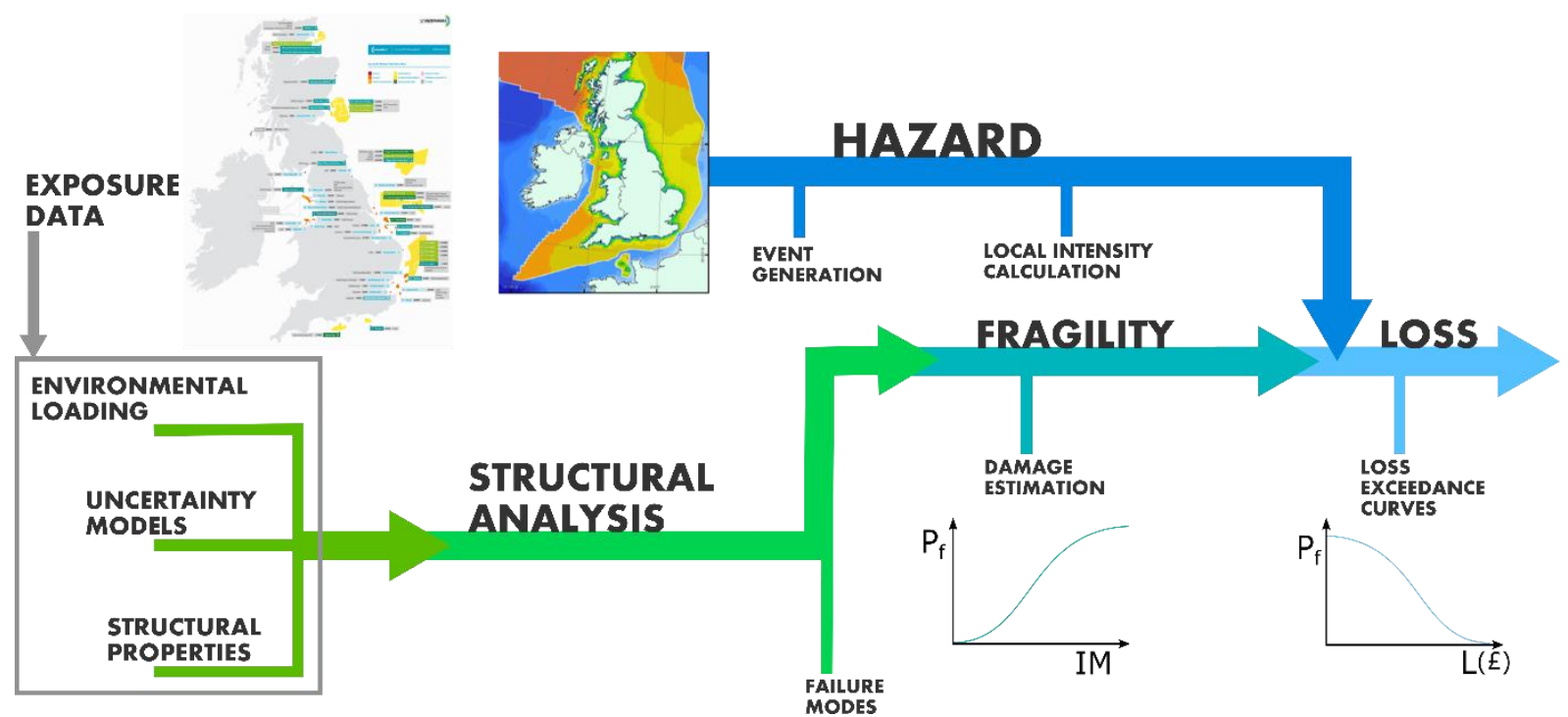

FIGURE 2. Probabilistic risk modelling framework for a single OWT structure. 


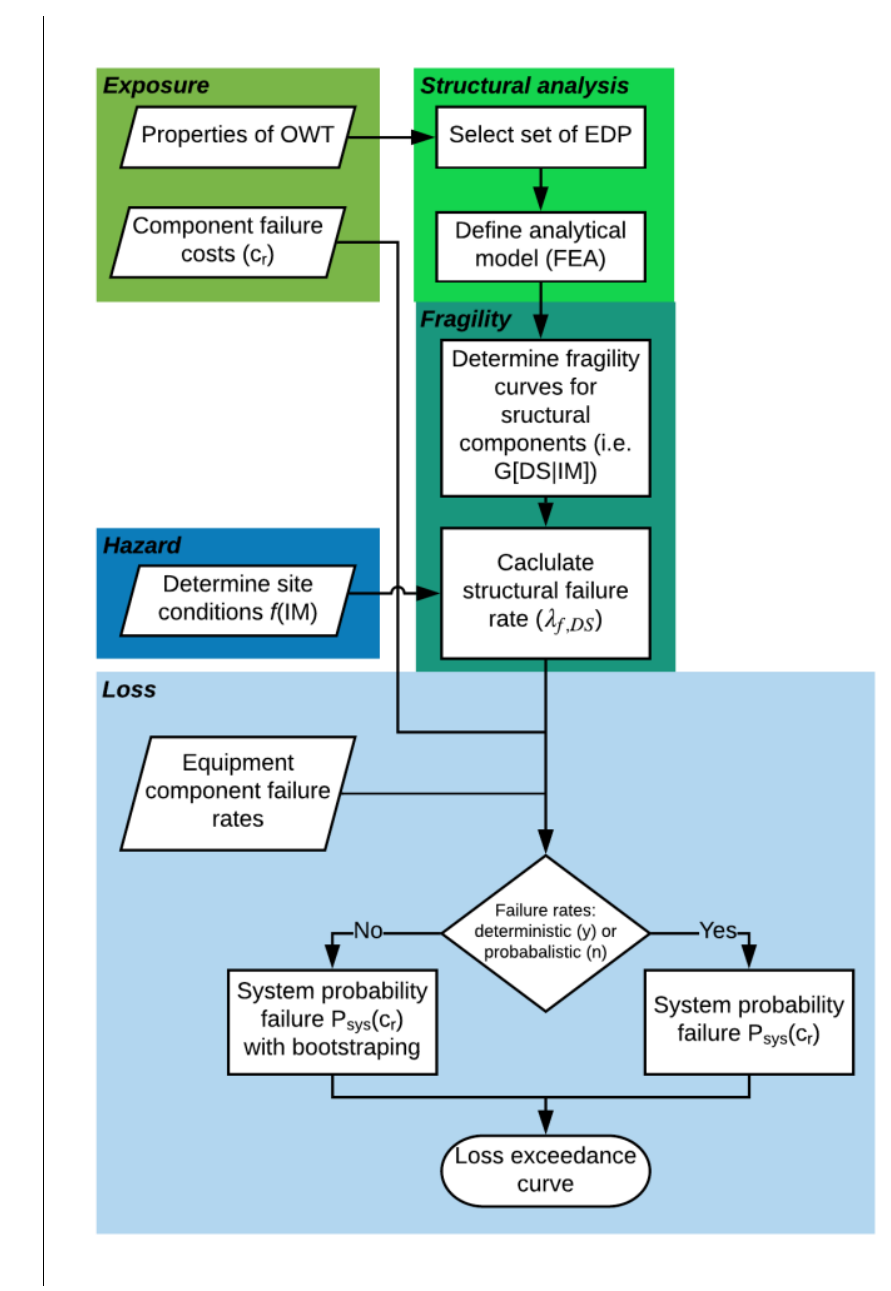

FIGURE 3. Proposed probabilistic risk modelling methodology used for the calculation of financial losses in this study.

\subsection{Hazard modelling}

The primary hazards threatening an OWT are those relating to severe wind and/or wave conditions. Wind and wave conditions are frequently parameterized using a separate variable to describe the severity of each (this is further discussed in Section 4.2). Common choices are the significant waves height (i.e., the average trough to crest height of the highest one-third waves [20]) and mean wind speed (with a 10-minute averaging period). The variables can be combined into a single IM by assuming that wind and waves are linked through the mean return period (MRP), i.e. the most extreme conditions associated with each MRP are coincident, a conservative, yet practical assumption used to assess OWTs [21]. This aspect is further discussed below.

Specific values of mean wind speed and significant wave height can be calculated using an appropriate probabilistic model, which describes frequency of occurrence of the environmental conditions at a site. Seismic hazards are also relevant for some sites [22], but are considered outside of the scope of this study.

\subsection{Exposure modelling}

The structural response of an OWT is highly dependent on turbine-specific parameters including the power rating and control system [23]. Consequently, exposure data has to be more 
detailed than that typically used in a probabilistic risk model for buildings, where only general information (e.g., in terms of construction class, height, and age of construction) about a portfolio of assets may be included [24]. Exposure data should include all pertinent information required to compute loss of the structure being assessed, this includes: location, geometric characteristics, material properties, and failure costs (i.e., replacement costs) for the OWT components.

\subsection{Structural analysis}

This step involves building a computational model solved using finite-element analysis, which predicts the response of the OWT to environmental conditions (wind and waves) represented through the selected IMs. For an OWT exposed to stochastic environmental loading, the use of dynamic (time-history) analysis is a common approach [23]. A first step consists of specifying a set of Engineering Demand Parameters (EDPs) representing the response of structural components of interest and computed through structural analysis. A key element of this process consisting of specifying a set of limit (or damage) states (DSs) defining structural performance criteria. Violation of a limit state indicates (conventional) failure of the structure [25] and these conditions are usually formulated as an equation containing a capacity (or resistance) and a demand (or load) term. In the ultimate limit state (ULS), for instance, failure of an OWT relates to the exceedance of the structure load-carrying capacity [26], where the demand is a function of the forces caused by environmental loading and the capacity relates to the ability of the structure to withstand these loads. All the structural components are exposed to this form of failure and should therefore be assessed by an analytical model as empirical failure data is very scarce. This includes the tower, monopile, transition piece and blades; see [27] for a detailed discussion on the topic

\subsection{Fragility analysis}

Fragility functions express the probability that a damage state occurs (or is exceeded) for a level of hazard intensity (i.e., IM), typically as a conditional CDF, $G[D S \mid I M]$ [28]. The structural analysis model described previously is typically used to estimate the probability of failure conditional on the IM. This is achieved by running the simulation repeatedly over a discrete set of IM values, resulting in a set of analysis outputs corresponding to each realization of the IM. Scatter in the fragility function parameters is caused by different wind and wave time-series causing different structural loading, and the effect of other random variables modelling structural demand and capacity [27]. The probability of failure can be estimated as the mean number of structural analyses resulting in the exceedance of the considered limit state (i.e., failures) at each IM value. The probability of failure can then be expressed as a functional relationship either by fitting a parametric distribution (e.g., lognormal) or directly using the output from structural analysis to generate an empirical fragility curve (e.g. [29]).

To combine the structural and equipment component failure, the structural fragility curves have to be converted into a failure rate $\left(\lambda_{f, D S}\right)$, for the given damage state (DS), to be compatible with equipment failure data. One way to achieve this is to apply the total probability law:

$$
\lambda_{f, D S}=\int G[D S \mid I M] \cdot|d \lambda(I M)| \cong \sum_{i=1} G\left[D S \mid I M_{i}\right] \cdot\left(\frac{1}{M R P_{i}}-\frac{1}{M R P_{i+1}}\right)
$$

The term $d \lambda(I M)$ in Eq. (3) can be computed through the derivative of the hazard curve $(\lambda(I M))$; it can be approximated by converting each MRP into an annual rate of exceedance, as shown in Eq. (3) and summing over a discrete set of MRP values, indexed by $i$. The fragility curves are calculated using a limited sample of structural simulations, therefore are associated

Towards Resilient OWF: A Probabilistic Framework for OWT Loss Assessment 10/06/2019 
with uncertainty. The effect of this statistical error can be quantified by resampling the chosen consequence metric (defined in the following section) using bootstrapping [30], as further discussed below.

\subsection{Loss assessment}

As already discussed, an OWT consists of different components. In general, for a system with a number $(N)$ of independent components, each of which has two discrete states (failure or operation), there is a finite number of permutations in the system state, where the total number of combinations of component events (leading to system events) is $2^{N}$. These combinations of operating and failure states can be summarized in a matrix $K$ [31], with elements $k_{i j} \in \mathbb{Z}^{N \cdot 2^{N}}$, using one to indicate that the component fails or zero to indicate that it remains operational. For a generic OWT with 11 components (e.g. Table 1), the matrix $\boldsymbol{K}$ will have elements $k_{i j} \in$ $\mathbb{Z}^{11 \cdot 2048}$; the first column will read $\left[\begin{array}{llllllllll}0 & 0 & 0 & 0 & 0 & 0 & 0 & 0 & 0 & 0\end{array}\right]^{T}$ indicating the case in which all components are functional, and the last $\left[\begin{array}{llllllllllll}1 & 1 & 1 & 1 & 1 & 1 & 1 & 1 & 1 & 1 & 1\end{array}\right]^{T}$ indicating the case where all components have failed. The intermediate columns will contain all other permutations of ones and zeros for different system states.

If each component has a deterministic material cost, the discrete system failure events can be combined to assess the probability of incurring a total material cost $\left(c_{r}\right)$. The matrix of the failure events $\boldsymbol{K}$ is converted into a failure cost matrix $\boldsymbol{K}_{\boldsymbol{c}}$ by multiplying each column of $\boldsymbol{K}$ by a vector containing the material cost of each component. This new matrix will contain the same number of elements as $\boldsymbol{K}$ but the values will equal the material costs as opposed to a logical value ( 1 or 0$)$. Then, $P_{\text {sys }}\left(c_{r}\right)$ can be defined as the probability that a set of components $\boldsymbol{k}^{*} \in$ $\boldsymbol{K}_{\boldsymbol{c}}$ fail whose combined material cost is equal to the target $\left(c_{r}\right)$ :

$$
P_{s y s}\left(c_{r}\right)=\sum_{\boldsymbol{k}^{*} \in \boldsymbol{K}_{\boldsymbol{c}}} \prod_{i=1}^{N} P_{i}^{k_{i}}\left(1-P_{i}\right)^{1-k_{i}}
$$

$P_{\text {sys }}\left(c_{r}\right)$ is evaluated over all the columns of the $\boldsymbol{K}$ matrix where the total material cost of the components equals $c_{r}$, i.e., $\boldsymbol{k}^{*}$ is a subset of $\boldsymbol{K}$ containing all vectors of system status with an equal cost. Assuming statistical independence between the different components, the probability of each system material cost is the product of the individual component failure probabilities in the matrix of failure events $\boldsymbol{k}^{*}$. When the element of the $\boldsymbol{k}^{*}$ matrix, $k_{i}$, is 0 then the probability that the component survives is used, i.e., $\left(1-P_{i}\right)^{1-k_{i}}$; and if $k_{i}$ is 1 , then the probability that the component fails is used, i.e., $P_{i}^{k_{i}}$.

In Eq. (5), the overall failure consequence, $C$ (Eq. (1)) or total annual loss, can be calculated by multiplying the yearly probability of different failure costs occurring $\left(P_{s y s}\left(c_{r}\right)\right)$ by the failure consequence defined by direct material cost $\left(c_{r}\right)$ and summing over all failure costs:

$$
C_{\text {storms }}=\text { Loss }_{\text {total }}=\sum_{c_{r}} P_{\text {sys }}\left(c_{r}\right) \cdot c_{r}
$$

\section{$4 \quad$ Illustrative application}

\subsection{Case-study sites}

Two OWF locations, which have previously been used by the authors to derive fragility relationships for OWTs on monopile foundations [27], are investigated in this paper. These

Towards Resilient OWF: A Probabilistic Framework for OWT Loss Assessment 10/06/2019 
sites are located at Ijmuiden K13, off the coast of the Netherlands, and off the coast of Massachusetts (at $40.5^{\circ} \mathrm{N} 69.3^{\circ} \mathrm{W}$ ), USA. These locations are plotted in the inset graph in Figure 4 (left).

The water depth at both sites is around 20m, making them suitable locations for the NREL 5MW OWT on a monopile foundation. The main elevations of the structure are shown in Figure 4 (right). As indicated in the figure, the tower terminates at an elevation above mean sea level of $\sim 90 \mathrm{~m}$. A full list of dimensions and material properties of the turbine are provided by [32]. Uncertainties in the parameters defining the OWT and model uncertainties are described in [27].

The structural components included in this study are the tower and blades, based on previous work by the authors [27], as the monopile and transition piece were observed to always fail after the tower had reached $100 \%$ probability of failure. This indicates that the tower is predicted to always fail first. The replacement cost for the tower is estimated through a parametric equation described in Section 4.1.1. Data for the non-structural components of the OWT (and the replacement costs for the blades) are taken from the work of Carroll et al [33]. They analyzed data from maintenance records of $\sim 350$ OWTs ranging from $2 \mathrm{MW}$ to $4 \mathrm{MW}$ in European waters and presented the results for different sub-systems. In this work, we focus on severe failures associated with either major repairs or full component replacement, and not on routine maintenance tasks. Only the failure rates for the top nine components in terms of replacement cost (out of a total of 19 sub-systems) are used in this work and are shown in Table 1. The costs have been rounded to the nearest $€ 1,000$, to improve computational efficiency when evaluating Eq. (4) numerically, so that step sizes of $€ 1,000$ could be used.

The different failure conditions considered in this paper are shown in Figure 5, which presents the considered scenarios through an event-tree diagram. The equipment is lumped together, as the failure of these components is assumed to have no impact on the failure of other components. Based on Figure 5, it is assumed that if the blades fail, the loads on the tower reduce (blades are the main source of aerodynamic loading) and, as a consequence, the tower may survive. This simplifying assumption, further tested here, is based on failure observations of onshore wind turbines exposed to typhoon conditions [34]. However, it should be noted that a blade may impact the tower if it breaks first. If the blades survive but the tower collapses, it is assumed that all equipment and the blades fall into the sea and are damaged.

TABLE 1. Material cost for major replacement and failure rate of OWT components. ${ }^{1}$ Eq. (6) with data $-\left[P_{W T}=5 M W\right]$.

\begin{tabular}{clcc}
\hline $\begin{array}{c}\text { Source of } \\
\text { cost data }\end{array}$ & Component & $\begin{array}{c}\text { Major replacement } \\
{[\boldsymbol{\epsilon}]}\end{array}$ & $\begin{array}{c}\text { Failure rate } \\
\text { [/turbine/year] }\end{array}$ \\
\hline \multirow{6}{*}{ Gearbox } & 230,000 & 0.154 \\
& Hub & 95,000 & 0.001 \\
& Transformer & 70,000 & 0.001 \\
& Generator & 60,000 & 0.095 \\
Carroll & Circuit breaker & 14,000 & 0.002 \\
& Power supply & 13,000 & 0.005 \\
& Pitch system & 14,000 & 0.001 \\
& Yaw system & 13,000 & 0.001 \\
& Controller & 13,000 & 0.001 \\
& Blades (x3) & 270,000 & MA: $9.89 \cdot 10^{-4}$ \\
\hline
\end{tabular}

Towards Resilient OWF: A Probabilistic Framework for OWT Loss Assessment 


\begin{tabular}{clcc}
\hline $\begin{array}{c}\text { Parametric } \\
\text { equations }\end{array}$ & Tower & $770,000^{1}$ & $\begin{array}{c}\text { MA: } 4.97 \cdot 10^{-3} \\
\text { IJ: } 8.36 \cdot 10^{-5}\end{array}$ \\
\hline & Total cost & $1,562,000$ & \\
\hline
\end{tabular}
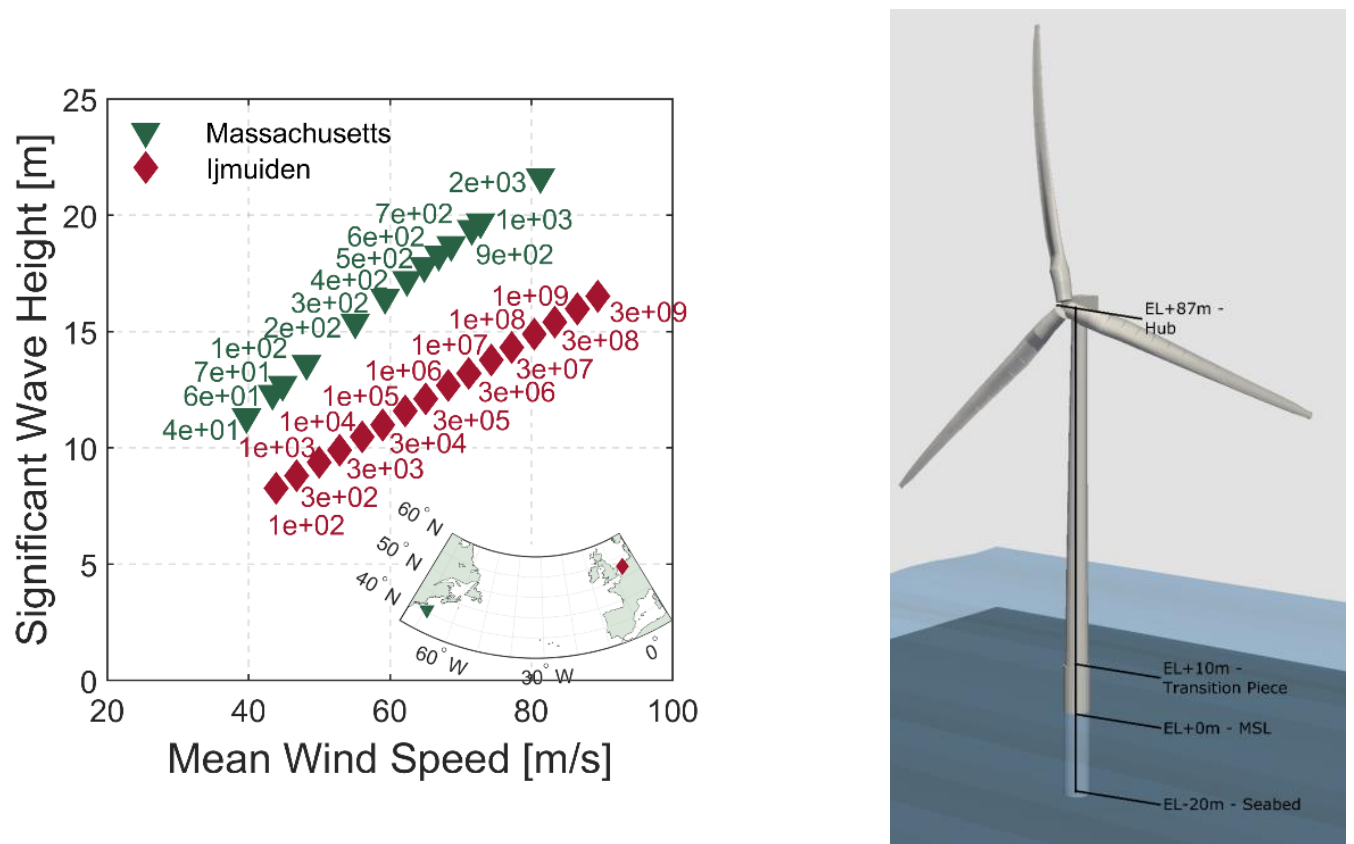

FIGURE 4. Comparison of the extreme wind and wave conditions associated with different MRPs at Massachusetts and Ijmuiden OWF sites (left) where the MRP is plotted beside the data points; the inset map shows the locations of both sites. Schematic figure of the case-study OWT used in this study, with main elevations highlighted (right).

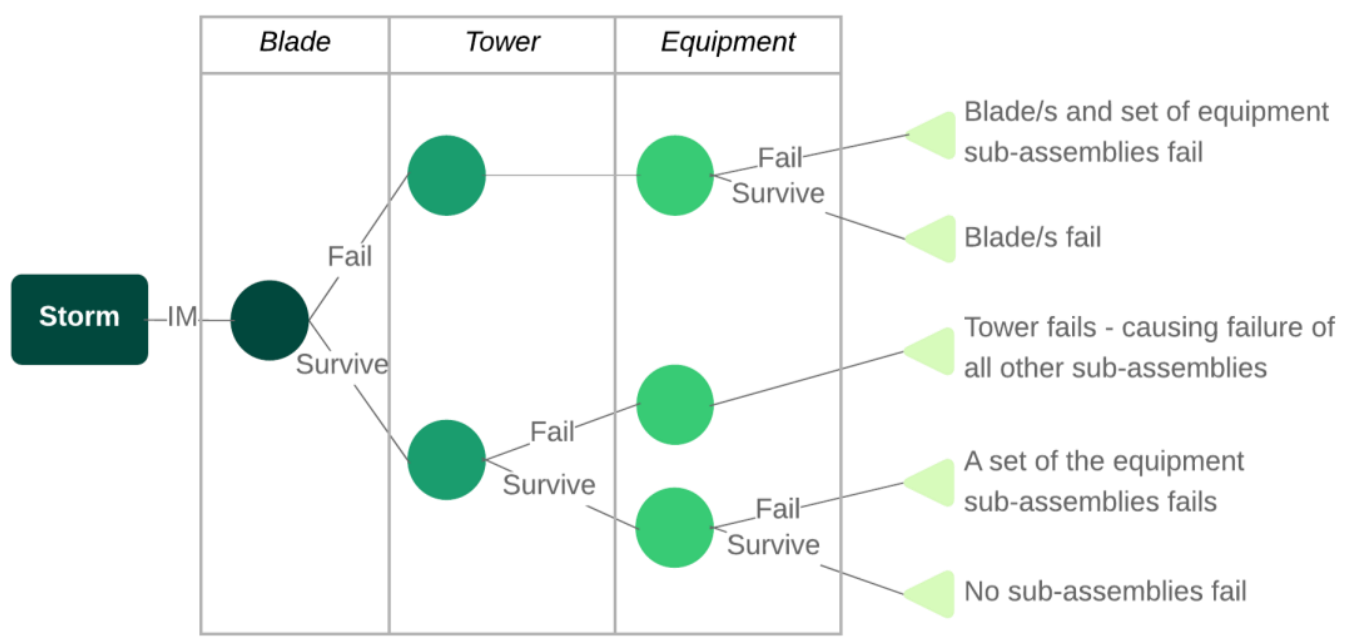

FIGURE 5. Event tree for an OWT combining structural and equipment components

\subsubsection{Structural failure cost}

Total OWT cost $\left(c_{W T}\right)$ in $\mathrm{k} €$, including blades and drivetrain but excluding foundations, is estimated using a parametric equation [35]. The independent variable in the equation is the rated power of the turbine $\left(P_{W T}\right)$ in megawatts $(\mathrm{MW})$; and the equation predicts the total turbine Towards Resilient OWF: A Probabilistic Framework for OWT Loss Assessment 
cost at various power ratings, i.e., from 2MW through to 5MW. This was converted into Euros (from Pound Sterling) by Dicorato et al [36] resulting in:

$$
c_{W T}=2.95 \cdot 10^{3} \cdot \ln \left(P_{W T}\right)-375.2
$$

Analysis by the NREL [37] reported that cost of an onshore wind turbines tower comprised $17.6 \%$ of the total turbine cost. The cost of the OWT tower is calculated by factoring down the wind turbine cost to $17.6 \%$ of $c_{\mathrm{WT}}$, assuming consistency in the relative cost between onshore and OWT components. Information about the specific cost of OWT towers would improve this calculation.

\subsection{Hazard model}

As discussed above, the hazard intensity calculation is simplified in this paper by combining the wind and wave conditions into a scalar IM - the mean return period (MRP), as shown in Figure 4 (left). This approach is conservative [21] but simplified the analysis substantially, reducing the number of required structural simulations. However, Wei et al. [21] have found that using environmental conditions coupled through MRP values instead of their joint probability distributions has little impact on the computed probability of failure.

Mean wind speeds and significant wave heights are plotted against their corresponding MRPs, in Figure 4 (left); both are evaluated using site-measured data. Ijmuiden has 22 years' worth of wind and wave measurements [38], stored as 3-hour averaged data, and statistical model representing the occurrence of different mean wind speeds and significant wave heights [20] have been developed by applying linear regression with log correction to the recorded data using [38]:

$$
\begin{gathered}
H_{s}=0.479 \cdot \ln (M R P)+6.063 \\
V_{w}=2.645 \cdot \ln (\mathrm{MRP})+31.695
\end{gathered}
$$

In Eq. (7), $H_{s}$ is the predicted three-hour significant wave height in meters, and $V_{w}$ is the 10minutes hub-height mean wind speed in $\mathrm{m} / \mathrm{s}$ (10 minutes averaging is a standard assumption based on the observation that mean wind speed is approximately constant over this period of time). At the offshore Massachusetts site, hurricane conditions were estimated by Wei et al [39] by fitting a generalised extreme value (GEV) distribution to the output from a stochastic catalogue of simulated hurricanes over a 100,000 year period (typical length of stochastic catalogs in some probabilistic risk models). The GEV probability distribution $(f(x \mid k, \sigma, \mu))$ is defined:

$$
f(x \mid k, \sigma, \mu)=\frac{1}{\sigma} \cdot\left(1+k \cdot \frac{x-\mu}{\sigma}\right)^{(-1 / k)-1} \cdot \exp \left(-\left(1+k \cdot \frac{x-\mu}{\sigma}\right)^{1 / k}\right.
$$

In Eq. (8), the model parameters are shape $(k)$, scale $(\sigma)$ and location $(\mu)$; these are defined for the Massachusetts site using the values in Table 2. The $x$ term is a variable that represents either the mean wind speed or significant wave height, depending on which set of parameters are used.

Table 2: GEV parameters for the Massachusetts (MA) wind farm site [39].

\begin{tabular}{cccc}
\hline Parameter & Shape $(\boldsymbol{k})$ & Scale $(\boldsymbol{\sigma})$ & Location $(\boldsymbol{\mu})$ \\
\hline \hline $\mathrm{V}_{\mathrm{w}}$ & 0.092 & 6.290 & 12.226 \\
$\mathrm{H}_{\mathrm{s}}$ & 0.038 & 2.117 & 2.972 \\
\hline
\end{tabular}

Towards Resilient OWF: A Probabilistic Framework for OWT Loss Assessment $10 / 06 / 2019$ 


\subsection{Structural analysis}

Structural analysis is based on dynamic (time-history) simulations with integrated wind and wave loading. The sea-state is modelled as a random process by using a wave spectrum, representing the frequency content of the sea-state, and is converted into a time-history using the inverse Fourier transform [20]. Similarly, the turbulent wind acting on the OWT is continuous, but is commonly evaluated numerically at discrete points on a grid overlying the structure. The turbulent wind time histories are evaluated using Turbsim [40], which converts a Kaimal spectrum into a stochastic time-history using inverse Fourier transforms. Dynamic response of the structure to this loading is calculated using the aeroelastic computer-aided engineering software Fatigue Aerodynamics Structures and Turbulence (FAST) [41] to run sets of time-history analyses. The wave theory used in the software is linear, which under predicts the peak wave loads of large storm waves. This simplification can be overcome by inserting non-linear waves replacing large waves, poorly assessed by the linear wave theory, for instance, by using the approach proposed by Hallowell at al [42]. Given the illustrative nature of the application presented here, it is decided to just rely on linear wave kinematics as implemented in FAST. Therefore, specific numerical results from this study should be regarded with caution.

Within FAST, the tower, monopile and blades are modelled as Euler-Bernoulli beams. No below seabed foundation is included within the structural analysis. Full details of the analysis methodology and the procedure for modelling stochastic wind and wave conditions is described in [27].

The structural limit states considered in this study is the ULS, including failure of the blades and tower, as discussed in more detail in Wilkie and Galasso [27]. Specifically, the tower limit state $\left(G_{M c r}\right)$ is taken from the work of Sorenson et al. [14]:

$$
\begin{gathered}
G_{M c r}=\frac{1}{6}\left(1-0.84 \cdot \frac{D}{t} \cdot \frac{F_{y}}{E}\right)\left(D^{3}-(D-2 t)^{3}\right) X_{m a t} X_{c r} F_{y}- \\
M_{U L T}\left(X_{a z}, X_{y a w}\right) X_{d y n} X_{s i m} X_{s t r}
\end{gathered}
$$

In Eq. (9), $D$ is the component diameter (m), $t$ is the thickness (m). The material properties are modelled as random variables through the yield stress $\left(F_{y}\right)$ and the Young's modulus $(E)$. $M_{U L T}\left(H_{s}, V_{w}\right)$ is the EDP selected and is defined as the maximum bending moment in the structural analysis depending on the mean wind speed $\left(V_{w}\right)$ and significant wave height $\left(H_{s}\right)$. Additionally, the $X$ terms are variables which capture modelling uncertainty including: structural dynamics $\left(X_{d y n}\right)$, simulation statistics $\left(X_{\text {sim }}\right)$, critical load capacity $\left(X_{c r}\right)$, material model uncertainty $\left(X_{\text {mat }}\right)$. The distributions used to model the random variables are described in Wilkie and Galasso [27].

A blade limit state was used $\left(G_{b l d}\right)$, using the simplifying assumption that failure can be predicted based on the maximum root flapwise moment. The limit state is defined as the blade flapwise moment capacity $\left(M_{c a p}\right)$ minus the blade flapwise moment demand $\left(M_{d e m}\right)$ :

$$
G_{b l d}=M_{c a p}-M_{d e m}\left(X_{a z}, X_{y a w}\right) \cdot X_{d y n} X_{\delta l} X_{s t r}
$$

The flapwise moment capacity is taken as $15,310 \mathrm{kNm}$ and was calculated by Resor [43] using FEA analysis for the NREL 5MW OWT blades. The additional modelling uncertainty is blade model uncertainty $\left(X_{\delta l}\right)$. Also in this case, the distributions and statistics used to model the random variables in Eq. (10) are described in Wilkie and Galasso [27].

Towards Resilient OWF: A Probabilistic Framework for OWT Loss Assessment $10 / 06 / 2019$ 


\section{$5 \quad$ Results and discussion}

\subsection{Fragility curves}

The fragility curves used in this study were developed by the authors [27], by selecting 16 MRPs and running a total of 400 structural simulations $\left(N_{A n}\right)$ at each MRP to calculate the probability of failure given the MRP. This analysis includes a set of random variables (referred to as X3 in the reference, as discussed in Section 4.3) which modelled variability in material properties, model uncertainty and orientation of the blades with respect to the incoming wind flow (the $X_{a z}$ variable in Eq. (9) and Eq. (10)). The tower fragility is represented by a parametric lognormal distribution and the blade fragility by using an empirical cumulative distribution function (as the lognormal assumption was found to be a poor fit to the analysis outputs). These relationships are shown in Figure 6 for both sites. The large difference between the IM-axis scales at the two sites is due to the hurricane conditions which are experienced at the MA site, making failure occurring at much lower MRP value than at the IJ site.

The fragility calculation was implemented by assigning an indicator function to the output from structural analyses, run at each MRP. When the limit state $(G)$ was violated i.e., $I(G \leq 0)$, a value of one indicated that the structural component (tower or blade) failed during the simulation. A fragility function was fit to this discrete data and provided a continuous prediction of the probability of failure conditional on the IM. In the case of the tower, maximum likelihood estimation (MLE) was used to predict best-fit parameters of the lognormal distribution, the lognormal mean $\mu_{L N}$ and lognormal standard deviation $\sigma_{L N}$. In the case of the blades, an empirical fragility curve (non-parametric) was derived by using previous neighbor interpolation between the 16 MRP analyzed.

The results in the original study were based on estimating the fragility function parameters through MLE based on a sample of structural simulations. This estimation is naturally associated with statistical error, and therefore there is uncertainty in the prediction of fragility function parameters, i.e. they will vary depending on the sample. For this reason, a large number of samples were used in the original paper (a single fragility curve requiring $N_{M R P} \cdot N_{A n}=$ 6,400 simulations). This is a fairly large number for many practical applications and, therefore, this study uses statistical resampling to assess the error introduced by using a reduced structural simulation size. Epistemic uncertainty is assessed by sampling new sets of fragility data, with replacement, from the original set of analysis results. The impact of the uncertainty caused by a smaller sample size can be evaluated by quantifying the scatter in failure rates resulting from scatter in the fragility curve parameters.

The original set of indicator functions at each IM (size $N_{A n}$ ) can be resampled with replacement for new, reduced number of analyses $\left(N_{A n, R e d}\right)$. The impact of analysis sample size can then be evaluated by quantifying the scatter in the failure rate that results from scatter in the fragility curve parameters. An example of the variability in the fragility curves is shown in Figure 7 for the OWT blades at the MA site, comparing the original $N_{A n}$ of 400 to a $N_{A n, R e d}$ of 50 . It can be observed that for the larger sample, size the scatter in the curves is reduced, however the mean prediction (bold black line) does not vary significantly. 

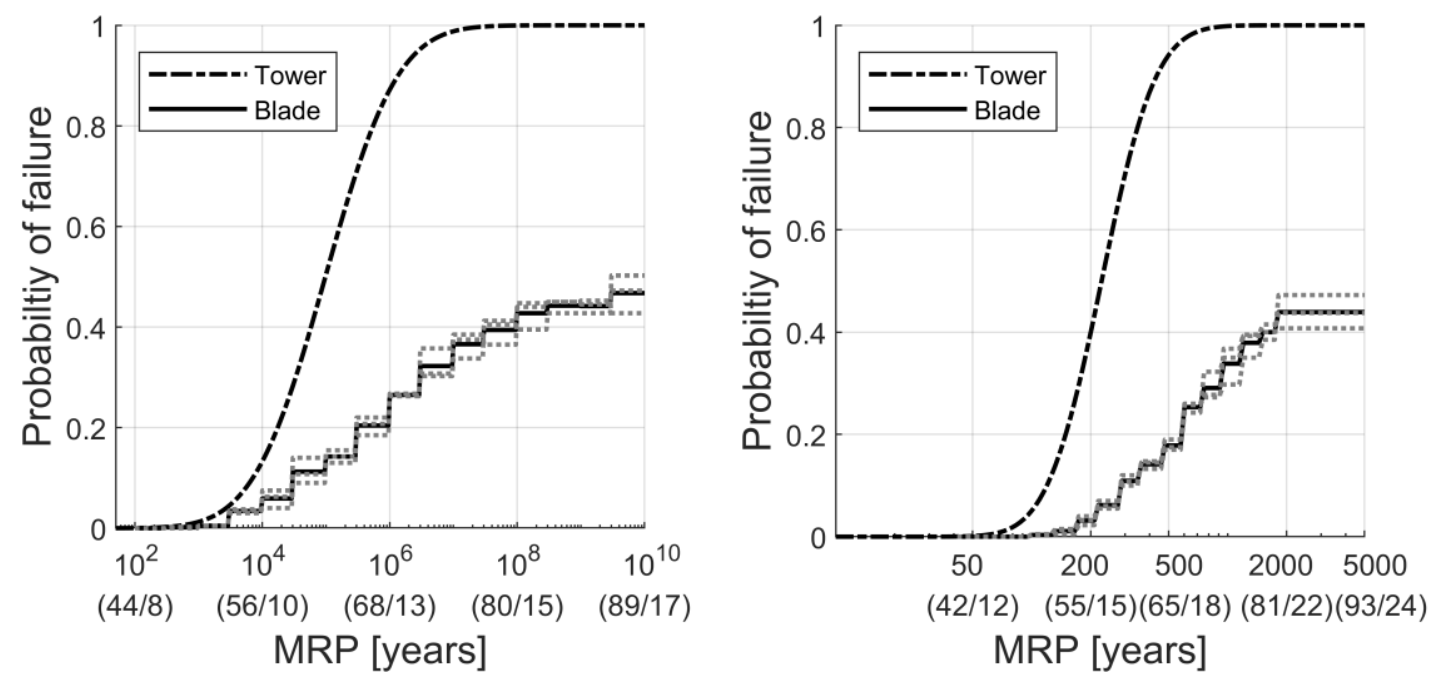

FIGURE 6. Fragility curves for the tower and blade at the IJ (left) and MA (right) site. The grey lines indicate the empirical fragility curves for individual blades and the black line indicates the expectation over the three blades. Brackets on the $\mathrm{x}$-axis labels contain $\left(\mathrm{V}_{\mathrm{w}}(\mathrm{m} / \mathrm{s}) / \mathrm{H}_{\mathrm{s}}(\mathrm{m})\right)$.
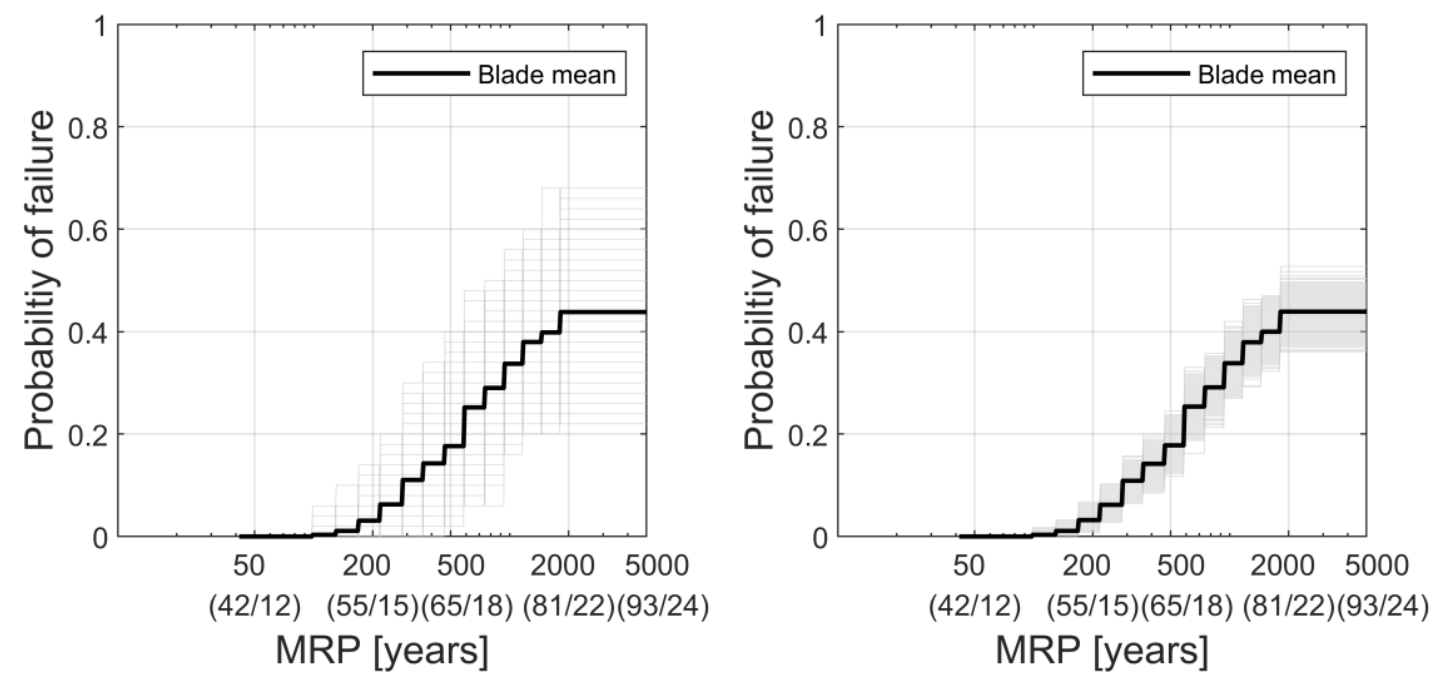

FIGURE 7. Resampled fragility curves shown in grey for the blades at the MA site. The number of samples used to calculate the probability of failure at discrete IM is 50 (left) and 400 (right). Brackets on the $\mathrm{x}$-axis labels contain $\left(\mathrm{V}_{\mathrm{w}}(\mathrm{m} / \mathrm{s}) / \mathrm{H}_{\mathrm{s}}(\mathrm{m})\right)$.

\subsection{Structural component yearly failure rates}

The integral in Eq. (3) is solved numerically, over the range of MRP values bounded by the limits $M R P_{i}=\left[10,10^{6}\right]$ and using a step size of 1 year. The failure rate is then calculated using each of the resampled fragility functions (over a set of $50 \geq N_{A n, R e d} \geq 400$ ), resulting in scatter in the fragility function parameters. The mean annual failure rates are presented in Table 1, and the individual samples through histograms in Figure 8 for the IJ site and in Figure 9 for the MA site, using a small subset of the $N_{A n \text {,Red }}$ sampled. These figures show the reduced scatter as the number of samples increases. The $N_{A n \text {,Red }}$ sample size of 100 is used in remainder of this work (by fitting a lognormal distribution to the histogram using MLE) as the maximum coefficient of variation is around $0.5 \%$ (for the IJ tower). 

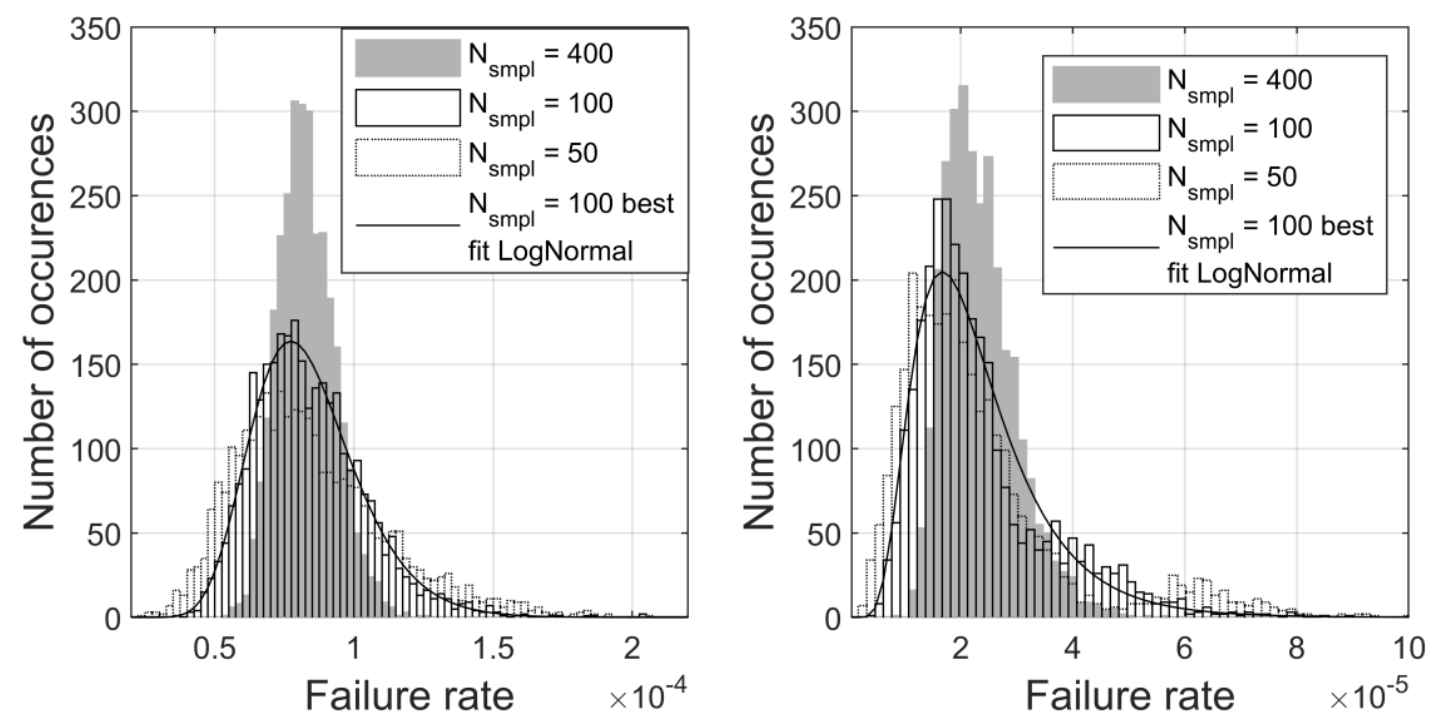

FIGURE 8. Histograms of failure occurrences for tower (left) and blades (right) for the IJ site.
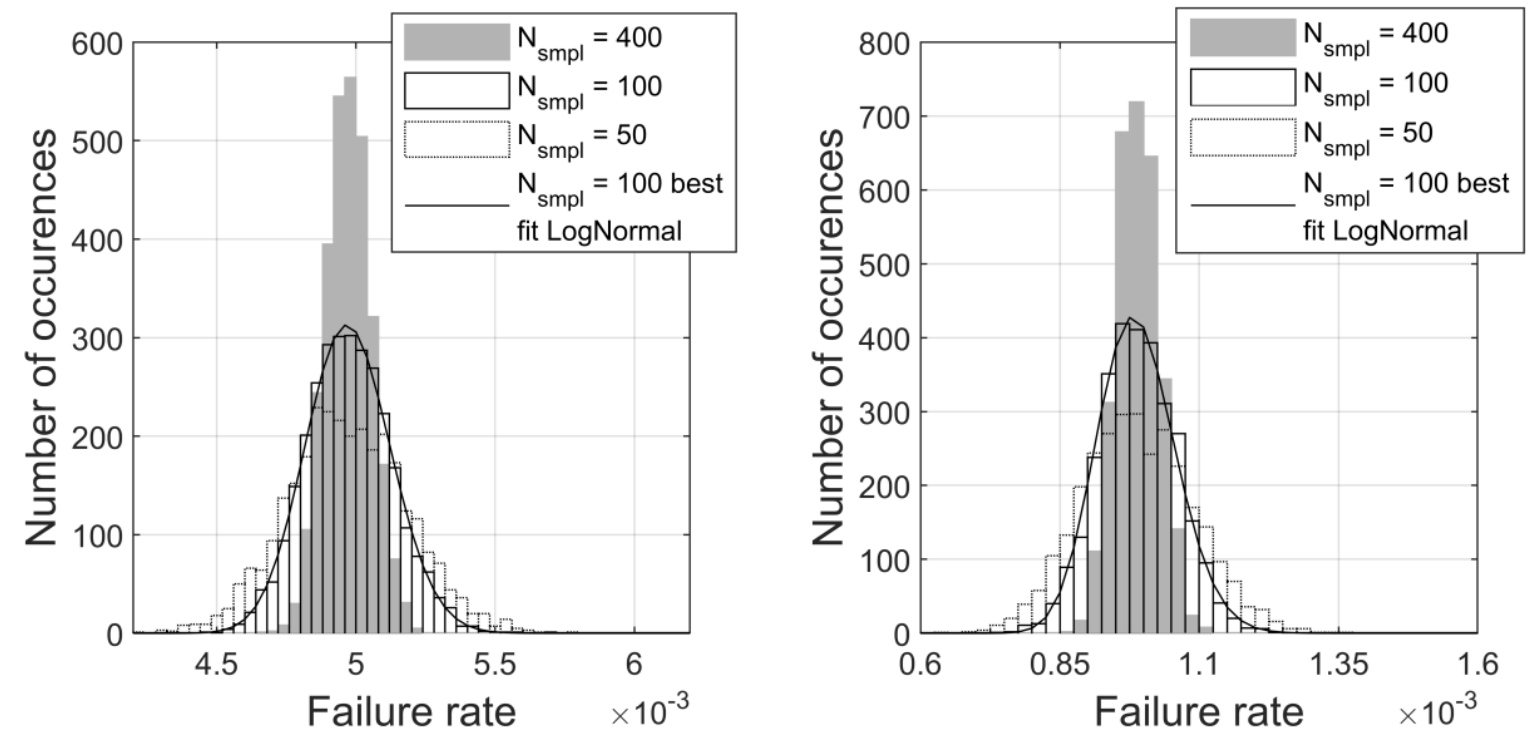

FIGURE 9. Histograms of failure occurrences for tower (left) and blades (right) for the MA site.

\subsection{Combined loss assessment}

Loss estimation is initially implemented using the mean failure rates from Table 1 . As discussed in Section 4, if the blades fail, loading pattern on the OWT will change (as the blades are the main source of aerodynamic loading). A simplified interpretation of this event is that, if the blades fail, the tower will be protected from failure through reduced loading. Conversely, if the blades survive but the tower collapses, it is assumed that all equipment and the blades fall into the sea and are damaged. To gain insight into the impact of these possible failure scenarios, four assumptions relating to failure of the OWT components are tested here:

- Case 1: Tower and other failures are perfectly correlated - A new matrix $\boldsymbol{K}_{1}$ is generated where failure of the tower results in the failure of all other components.

- Case 2: Blade failure prevents failure of the tower - A new matrix $\boldsymbol{K}_{2}$ is generated. Firstly, failure events that include the blades modified to prevent failure of the tower. 
Then the remaining cases where the tower fails (but the blades do not) cause failure of all other components.

- Case 3: Uncorrelated components - the $\boldsymbol{K}$ matrix left unchanged.

- Case 4: No structural failure, only equipment components fail, and empirical failure rates from Table 1 are used.

These assumptions about the dependency of the OWT components are encoded within the loss calculation by creating an updated matrix of failure events $(\boldsymbol{K})$. The updated matrix is used to evaluate which subset of failure events are used $\boldsymbol{k}^{*} \in \boldsymbol{K}_{\boldsymbol{c}}$ at each cost level in Eq. (4). Events that include failure of the tower can be identified, and the indices relating to all other components modified to correspond to a failure state. In the case where blade failure prevents failure of the tower, events where the tower and blades fail are first identified - then the tower component index is changed so that it survives. In the equipment only case, the probability of failure of the structural components is set to zero.

The annual loss CCDF is shown in Figure 10 (left) for the IJ site and (right) for the MA site. These show that losses of individual OWTs occur with relatively large probability driven by the more frequently occurring equipment failures, with annual probability of occurrence around $22 \%$ at both sites. Losses that also include structural components occur with annual probability of around $0.008 \%$ at IJ and $0.5 \%$ at MA, indicating the large difference in hazard.

The failure case which excludes structural components cannot predict material costs above $€ 1 \mathrm{M}$, all of which include the tower. Using independent components (Case 3 ) results in a range of failure costs that involve the tower, whereas the correlated failure cases only predicts a material cost that is the sum of all equipment and tower costs, $\$ 1,562,000$ (Table 1). This is more accurate, as collapse of the tower will have consequences for all equipment in the hub. Differences in assumptions about blade failure (Case 1 and 2) do not make a visible impact, due to rarity of blade failures in comparison to the tower at both sites. This is explained by Eq. (4), as for each set of failure events the yearly probability of occurrence is the product of the probability of failures (for components that fail) and probability of survival (for components that survive).
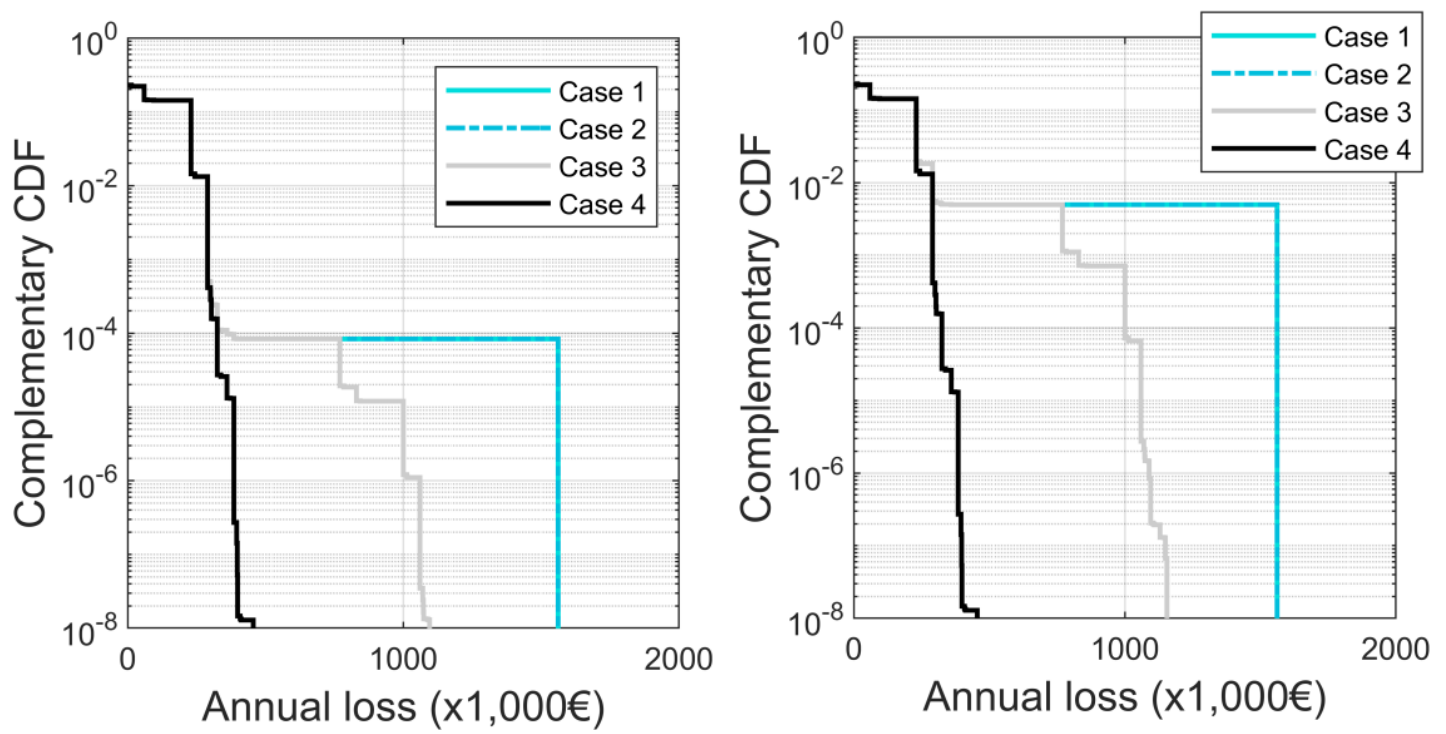

FIGURE 10. Loss CCDF comparing the four assumptions used in calculating loss at the IJ site (left) and MA site (right).

Towards Resilient OWF: A Probabilistic Framework for OWT Loss Assessment $10 / 06 / 2019$ 


\subsection{Annual loss distribution}

Loss for the OWT is estimated using Eq. (5). Uncertainty in the structural failure rates can be included in the loss calculation by sampling the distributions describing failure rates of the blades and tower (i.e., Figure 8 and Figure 9) and using the random samples as input to Eq. (3). The resulting distribution of annual losses is shown in Figure 11.

For IJ, little difference is visible in the two cases where perfect correlation in the failure cases is assumed; the mean annual loss of both is $€ 3.87 \mathrm{e} 4$. The uncorrelated case is not conservative, because the mean annual losses are lower; however the difference is only marginal as the mean Case 3 annual loss is $€ 3.86 \mathrm{e} 4$. Results for the MA site, Figure 12 (right), indicate that the structural components have a more important impact on annual loss. The Case 1 and 2 histograms both have mean annual loss of $€ 4.62 \mathrm{e} 4$, whereas the Case 3 (independent results) has a lower mean annual loss of $€ 4.25 \mathrm{e} 6$. This is due to the hurricane type conditions at the site, these differences emphasize the need for a site-specific approach to the structural components of OWT. The specific numerical results should be considered with caution, given the simplifying assumptions used in the illustrative application. However, the relative importance of the different analysis cases suggests that Case 1 provides a conservative estimation of annual losses.
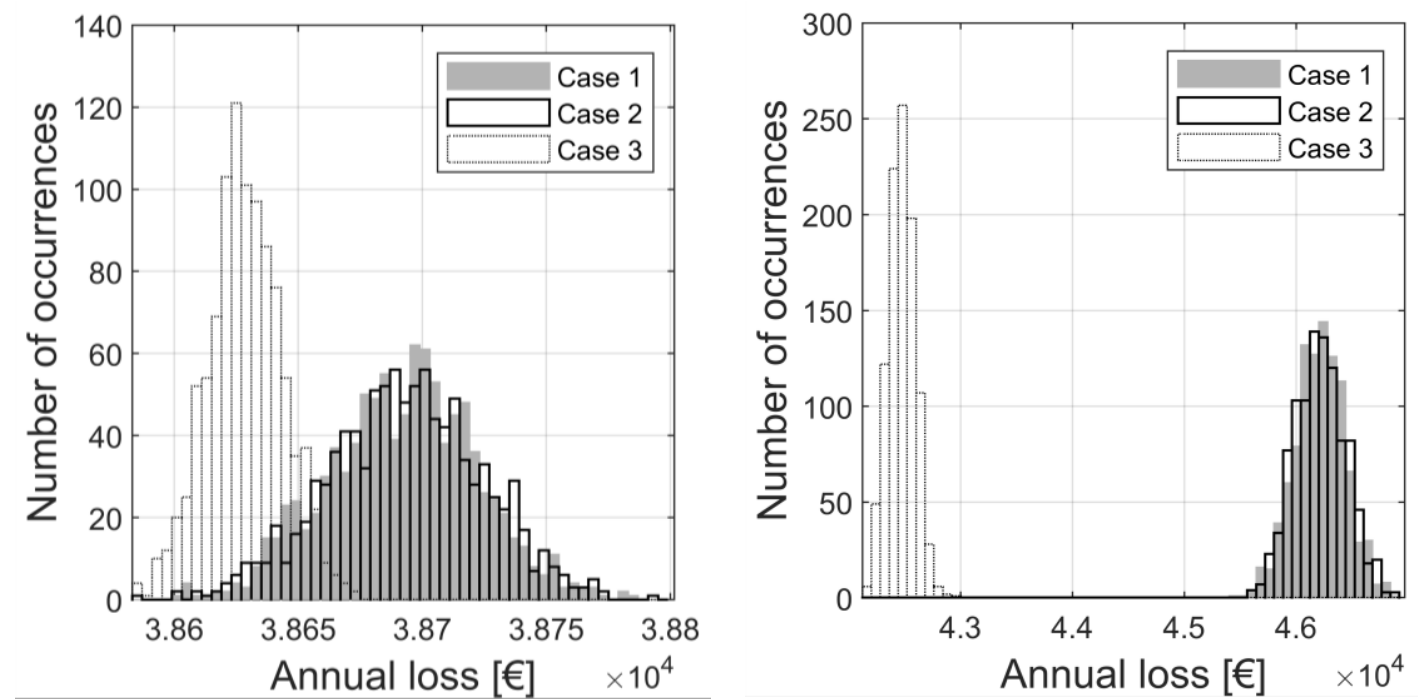

FIGURE 11. Histogram of annual loss for IJ (left) and MA (right) when uncertainty in the fragility function parameters is modeled.

\section{Conclusion}

This paper proposed a method for estimating structural robustness of an OWT, as a first step towards quantifying resilience of OWFs, by applying a probabilistic risk modelling framework to quantify financial losses due to extreme environmental conditions. This approach has been applied to a wide range of similar problems in civil engineering and therefore has a strong basis. However, it has never been used to estimate losses for an OWT, comprised of structural and mechanical / electrical components. An illustrative application demonstrated how the calculation could be implemented to estimate potential loss associated with the most important sub-systems of individual OWTs for two sites. Resilience is simplified here to the estimation of the consequence of OWT failure, which is defined in terms of material cost alone. This allowed the idea of resilience to be applied by practicing engineers who will not have access to data required for a full evaluation of resilience, including potential recovery phases. As robustness is a component of a full resilience calculation, the simplified method presented in this paper could be used as an input to a more comprehensive resilience assessment.

Towards Resilient OWF: A Probabilistic Framework for OWT Loss Assessment $10 / 06 / 2019$ 
The case-study assessed a turbine comprised of both generation equipment and structural components. Although structural failure rates were low in comparison to the mechanical components, they are associated with very high material costs. For this reason, they were found to have a notable impact on the annual loss at the MA site, where severe hurricane conditions occur. This highlights the site specific nature of the structure and emphasizes the need to include details of site loading into risk calculation, fragility will vary between sites [27]. It is noted that the case study presented here did not include the fatigue limit state, considering only ULS failure, in practice it would be feasible to assess both limit states within the framework presented. However, focusing only on material costs means that the specific numerical results should be interpreted with caution, as neglecting operational costs (such as vessel deployment) may skew the computed losses.

Future steps will involve considering the risk posed to an array or whole OWF in greater detail, due to correlated hazards i.e. a wind storm effects the whole installation simultaneously. If the framework is to be applied in practice the simplifying assumptions in the walk-through example would need to be mitigated through a more complex structural model. Additionally, the framework should also encompass the operational load-cases which may contribute significantly to the failure of the blade components.

Many challenges remain to be answered, particularly relating to the choice of performance indicators [13]. It is worth noting that validation is an important step to implement the proposed framework with confidence in many practical applications. However, structural failure cannot be validated through empirical data because it is characterized by very low probability of occurrence, as shown in the paper. The proposed framework relies on computer-based simulations; each component of this computer-based simulation has been validated in past studies. This includes, for instance, verification between different OWT analysis package, see Passon et al. [44]. However, if successful, this approach will aid in the development of integrated design techniques for OWTs and OWFs.

\section{$7 \quad$ Acknowledgements}

This work was supported by the UK Engineering and Physical Sciences Research Council (EPSRC), DTP grant EP/M507970/1 for University College London.

\section{$8 \quad$ References}

[1] Ho A, Mbistrova A. The European offshore wind industry - key trends and statistics 2016. 2017.

[2] Ho A, Mbistrova A. The European offshore wind industry - key trends and statistics 1st half 2016. 2016.

[3] Arwas P, Charlesworth D. Offshore wind cost reduction: pathways study. 2012.

[4] Van Der Tempel J. Design of support structures for offshore wind turbines. Technical University of Delft, 2006.

[5] Corbetta G, Mbistrova A, Ho A. Wind in power - 2015 European statistics. 2016.

[6] Bruneau M, Chang SE, Eguchi RT, Lee GC, O'Rourke TD, Reinhorn AM, et al. A framework to quantitatively assess and enhance the seismic resilience of communities. Earthq Spectra 2003;19:733-52. doi:10.1193/1.1623497.

[7] Hosseini S, Barker K, Ramirez-Marquez JE. A review of definitions and measures of system resilience. Reliab Eng Syst Saf 2016;145:47-61. doi:10.1016/j.ress.2015.08.006.

[8] Ayyub BM. Systems resilience for multihazard environments: Definition, metrics, and valuation for decision making. Risk Anal 2014;34:340-55. doi:10.1111/risa.12093.

[9] Ghosn M, Duenas-Osorio L, Frangopol DM, McAllister TP, Bocchini P, Manuel L, et

Towards Resilient OWF: A Probabilistic Framework for OWT Loss Assessment 
al. Performance indicators for structural systems and infrastructure networks. J Struct Eng 2016;142:F4016003. doi:10.1061/(ASCE)ST.1943-541X.0001542.

[10] Bocchini P, Frangopol DM, Ummenhofer T, Zinke T, Asce M, Frangopol DM, et al. Resilience and sustainability of civil infrastructure: toward a unified approach. J Infrastruct Syst 2014;20:1-16. doi:10.1061/(ASCE)IS.1943-555X.0000177.

[11] Cimellaro GP, Reinhorn AM, Bruneau M. Seismic resilience of a hospital system. Struct Infrastruct Eng 2010;6:127-44. doi:10.1080/15732470802663847.

[12] Bruneau M, Reinhorn A. Exploring the concept of seismic resilience for acute care facilities. Earthq Spectra 2007;23:41-62. doi:10.1193/1.2431396.

[13] Quiel SE, Marjanishvili SM, Katz BP. Performance-based framework for quantifying structural resilience to blast-induced damage. J Struct Eng 2015;142:C4015004. doi:10.1061/(ASCE)ST.1943-541X.0001310.

[14] Sorensen JD, Toft HS. Probabilistic design of wind turbines. Energies 2010;3:241-57. doi:10.3390/en3020241.

[15] Rose S, Jaramillo P, Small MJ, Apt J. Quantifying the Hurricane Catastrophe Risk to Offshore Wind Power. Risk Anal 2013;33:2126-41. doi:10.1111/risa.12085.

[16] Porter KA. An overview of PEER's performance-based earthquake engineering methodology. 9th Int Conf Appl Stat Probab Civ Eng 2003;273:973-80.

[17] Petrini F. A probabilistic approach to performance-based wind engineering (PBWE). Università degli Studi di Roma, 2009.

[18] Barbato M, Petrini F, Unnikrishnan VU, Ciampoli M. Performance-based hurricane engineering (PBHE) framework. Struct Saf 2013;45:24-35. doi:10.1016/j.strusafe.2013.07.002.

[19] Attary N, Unnikrishnan VU, van de Lindt JW, Cox DT, Barbosa AR. PerformanceBased Tsunami Engineering methodology for risk assessment of structures. Eng Struct 2017;141:676-86. doi:10.1016/j.engstruct.2017.03.071.

[20] DNV GL. DNVGL-RP-C205. Environmental conditions and environmental loads. 2017.

[21] Wei K, Arwade SR, Myers AT. Incremental wind-wave analysis of the structural capacity of offshore wind turbine support structures under extreme loading. Eng Struct 2014;79:58-69. doi:10.1016/j.engstruct.2014.08.010.

[22] De Risi R, Bhattacharya S, Goda K. Seismic performance assessment of monopilesupported offshore wind turbines using unscaled natural earthquake records. Soil Dyn Earthq Eng 2018;109:154-72. doi:10.1016/j.soildyn.2018.03.015.

[23] Vorpahl F, Schwarze H, Fischer T, Seidel M, Jonkman J. Offshore wind turbine environment, loads, simulation, and design. Wiley Interdiscip Rev Energy Environ 2013;2:548-70. doi:10.1002/wene.52.

[24] Mitchell-Wallace K, Jones M, Hillier J, Foote M. Natural catastrophe risk management and modelling. John Wiley \& Sons; 2017. doi:10.1002/9781118906057.

[25] Melchers RE, Beck AT. Structural reliability analysis and prediction. 3rd ed. John Wiley \& Sons; 2018. doi:10.1002/9781119266105.

[26] DNV GL. DNV-OS-J101. Design of offshore wind turbine structures. 2014.

[27] Wilkie D, Galasso C. Site-specific ultimate limit state fragility of offshore wind turbines on monopile substructures. Eng Struct [Under Rev n.d.

[28] Bakalis K, Vamvatsikos D. Seismic fragility functions via nonlinear response history analysis. J Struct Eng 2018;144:04018181. doi:10.1061/(ASCE)ST.1943541X.0002141.

[29] Iervolino I. Assessing uncertainty in estimation of seismic response for PBEE. Earthq Eng Struct Dyn 2017;46:1711-23. doi:10.1002/eqe.2883.

[30] Efron B, Tibshirani R. An introduction to the bootstrap. New York: Chapman \& Hall; 1993.

[31] Mensah AF, Dueñas-Osorio L. A closed-form technique for the reliability and risk assessment of wind turbine systems. Energies 2012;5:1734-50.

Towards Resilient OWF: A Probabilistic Framework for OWT Loss Assessment 
doi:10.3390/en5061734.

[32] Jonkman J, Butterfield S, Musial W, Scott G. Definition of a 5-MW reference wind turbine for offshore system development. 2009.

[33] Carroll J, McDonald A, McMillan D. Failure rate, repair time and unscheduled O\&M cost analysis of offshore wind turbines. Wind Energy 2016;19:1107-19. doi:10.1002/we. 1887.

[34] Chen X, Li C, Tang J. Structural integrity of wind turbines impacted by tropical cyclones: A case study from China. J. Phys. Conf. Ser., vol. 753, 2016. doi:10.1088/1742-6596/753/4/042003.

[35] Study of the costs of offshore wind generation; a report to the renewables advisory board \& DTI. 2007.

[36] Dicorato M, Forte G, Pisani M, Trovato M. Guidelines for assessment of investment cost for offshore wind generation. Renew Energy 2011;36:2043-51. doi:10.1016/j.renene.2011.01.003.

[37] Mone C, Smith A, Maples B, Hand M. 2013 Cost of wind energy review; NREL/TP5000-63267. 2015.

[38] Fischer T, de Vries W, Schmidt B. Upwind design basis - WP4. 2010.

[39] Wei K, Arwade SR, Myers AT, Hallowell S, Hajjar JF, Hines EM, et al. Toward performance-based evaluation for offshore wind turbine jacket support structures. Renew Energy 2016;97:709-21. doi:10.1016/j.renene.2016.06.028.

[40] Kelley ND, Jonkman B. NREL/TP-500-41137. Overview of the turbsim stochastic inflow turbulence simulator. 2007.

[41] Jonkman B, Jonkman J. FAST Readme v8.12.00a-bjj. Denver: 2015.

[42] Hallowell S, Myers AT. Site-specific variability of load extremes of offshore wind turbines exposed to hurricane risk and breaking waves. Wind Energy 2017;20:143-59. doi:10.1002/we. 1996.

[43] Resor BR. SAND2013-2569, Definition of a 5MW/61.5m wind turbine blade reference model. Albuquerque: 2013.

[44] Passon P, Kühn M, Butterfield S, Jonkman J, Camp T, Larsen TJ. OC3 - Benchmark Exercise of Aero-Elastic Offshore Wind Turbine Codes Preprint. EAWE Spec Top Conf Sci Mak Torque from Wind Univ Denmark, Lyngby, Denmark August 28-31, 2007 Conf Pap NREL/CP-500-41930 August, 2007 2007:15. doi:10.1088/17426596/75/1/012071. 